\begin{tabular}{|c|l|}
\hline Title & Directivity and apparent velocity of the coseismic ionospheric disturbances observed with a dense GPS array \\
\hline Author(s) & Heki, Kosuke; Ping, Jinsong \\
\hline Citation & $\begin{array}{l}\text { Earth and Planetary Science Letters, 236(3-4), 845-855 } \\
\text { https://loi.org/10.1016/.epsl.2005.06.010 }\end{array}$ \\
\hline Issue Date & $2005-08$-15 \\
\hline Doc URL & http://hdl.handle.net/2115/14616 \\
\hline Type & article (author version) \\
\hline File Information & EPSL2005-236-3-4.pdf \\
\hline
\end{tabular}

Instructions for use 


\title{
Directivity and apparent velocity of the coseismic ionospheric disturbances observed with a dense GPS array
}

\author{
*Kosuke Heki ${ }^{1}$ and Jinsong Ping ${ }^{2}$ \\ ${ }^{1}$ Division of Earth and Planetary Sciences, Hokkaido University \\ N10 W8, Kita-ku, Sapporo 060-0810, Japan \\ Tel: +81-11-706-3826 Fax: +81-11-746-2715, email: heki@ep.sci.hokudai.ac.jp \\ ${ }^{2}$ Shanghai Astronomical Observatory, Chinese Academy of Sciences, \\ 80 Nandan Rd., Shanghai 200030, China \\ Tel/Fax: +86-21-64876658 email:pjs@shao.ac.cn
}

\begin{abstract}
Coseismic disturbances in ionosphere have been observed after recent thrust earthquakes off the Pacific coast of Japan, with a dense Global Positioning System array. Positive pulses in electron content, as large as several times $10^{15}$ electrons $/ \mathrm{m}^{2}$ and as long as 4-5 minutes, emerge $\sim 10$ minutes after earthquakes and propagate horizontally with apparent velocity of $\sim 1 \mathrm{~km} / \mathrm{sec}$, close to the sound velocity at the ionospheric height. They may have been excited by coseismic uplifts of the sea surface initially as upward propagating compressive pulses of atmosphere, which were gradually refracted to propagate horizontally in ionosphere. The propagation has strong north-south asymmetry, and interaction between the movements of charged particles in acoustic waves and geomagnetic fields might have selectively attenuated the northward propagating disturbances.
\end{abstract}

Keywords: ionospheric disturbance, earthquake, TEC, GPS, Japan

\section{Introduction}

Ionospheric disturbances after large earthquakes have been found first with the Doppler sounding technique as the basal oscillation of the ionosphere [1,2]. Recently, Global Positioning System (GPS) has observed them as the perturbation in Total Electron Content (TEC), number of electrons integrated along the line-of-sights. Calais and Minster [3] first detected such a disturbance associated with the 1994 Northridge Earthquake, California. Recently, such a coseismic ionospheric disturbance (we refer to it as CID here) was observed with a dense GPS array in south California after the 2002 Denali Earthquake, Alaska [4]. However, there have been few CID studies with a dense GPS array close to epicenter.

In Japan, GPS Earth Observation Network (GEONET) has been in operation for over a decade with nearly one thousand continuous receiving stations, and contributed to crustal deformation studies as seen in recent review articles [5,6]. Here we present observations of CID associated with the 2003 September 25 (19:50:06 UT) Tokachi-oki Earthquake $\left(\mathrm{M}_{\mathrm{w}} 8.0\right)$, a typical interplate thrust earthquake at the Japan Trench near Hokkaido, and the foreshock $\left(\mathrm{M}_{\mathrm{w}} 6.9,10: 07\right.$ UT) of the 2004 September 5 Southeast Off-Kii-Peninsula Earthquakes, an earthquake with high-angle thrust mechanism occurred in the subducting Philippine Sea plate slab (we simply call them as the Tokachi earthquake and the Kii foreshock in this study). Fig.1 shows coseismic vertical crustal movements in these earthquakes, calculated following the Okada's [7] model with fault parameters reported by Tanioka et al. [8] and Earthquake Research Committee [9] for the Tokachi earthquake the Kii foreshock, respectively. In this study we take advantage of a dense array, and determine essential attributes of CID such as apparent propagation velocities, directionality, waveform, and initial motion, in order to establish a basic set of knowledge to study seismic wave propagation in the Earth's upper atmosphere.

\section{GPS data analysis}

Microwaves suffer from delays (time delay or phase advance) proportional to the inverse-square of the frequencies when they penetrate the ionosphere. GPS satellites transmit carriers in two frequencies in L-band (1.2 and $1.5 \mathrm{GHz})$ so that we can "remove" these delays by making their "ionosphere-free" linear 
combinations for accurate positioning purposes. On the other hand, we can also "isolate" ionospheric information by making the "ionospheric" linear combinations, simple differences between the two phases expressed in lengths. In this study we obtained the raw data (sampling interval 30 seconds) of $\sim 900$ GEONET sites of the days when earthquakes occurred from the ftp site of the Geographical Survey Institute (terras.gsi.go.jp). We converted the ionospheric combinations of the phases into TEC units (1 $\mathrm{TECU}=10^{16}$ electrons $/ \mathrm{m}^{2}$ ), and investigated the time series of individual satellites for CID signals. Linear combinations of pseudorange (code) data are often used as well as carrier phases, to obtain absolute TECs by removing ambiguities (and cycle-slips) in phase data. We did not do this here because we are only interested in short-term relative changes in TEC. Basic procedures in ionospheric studies with GPS are described in detail in past articles $[10,11]$.

Apparent TEC changes due to various reasons, such as diurnal variation and large-scale traveling ionospheric disturbances (LSTID). Band-pass filters, e.g. with a window 3-7 minutes [10], have been used in such studies, to isolate short-term disturbances. Here, in addition to this conventional approach, we also try polynomial fitting (a kind of high-pass filter) and wavelet transformation (a kind of band-path filter). In Fig. 2, we fit polynomials with degrees up to six and plot residuals to study CID waveforms. There, positive pulsations (or oscillations with positive initial motions) in TEC occurring 10-20 minutes after the earthquakes are the CID signals. Sub-ionospheric points (SIP), ground projections of points where line-of-sight vectors penetrate the ionosphere, were calculated assuming a thin ionosphere as high as $300 \mathrm{~km}$ [4]. SIPs are located within $500-600 \mathrm{~km}$ around the receiving sites, so it is possible that GPS points in southwestern Japan record CID signals of earthquakes in Hokkaido as will be shown later on. SIP tracks of the same satellite for different sites are approximated by simple translations (Fig.1b) but have slight distortions due to the Earth's curvature.

\section{Results}

\subsection{Waveform, period and initial motion}

For the Tokachi earthquake, CIDs were detected in signals of three satellites, 13, 24 and 27, whose SIP trajectories for the site "a" are plotted in Fig.1a. TEC time series for satellite 24 over the one-hour interval encompassing the earthquake are shown in terms of residuals from best-fit degree 6 polynomials (i.e. similar to 15 minutes high-pass filtered) in Fig.2a. Line-of-sight penetration angle corrections to convert TEC to vertical electron content are not applied in Fig.2. The lower and upper halves of Fig. 2a show signals observed at GPS sites in the Tohoku District and in Hokkaido (Fig. 1a), respectively. This satellite was very close to zenith during this time interval; the SIPs are close to the GPS sites. Disturbance signals move southward along the Tohoku District gradually changing their shapes. The signals of satellite 24 (Fig. 2a) look like a positive pulse (i.e. TEC increase) at site A. Then the secondary peak, $\sim 10$ minutes after the first one, seems to grow as it propagates along the arc.

The CID of satellite 13 (Fig. 2b) have a negative part and looks like an oscillation rather than a pulsation with the wavelength of 4-5 minutes. Such a period is close to the prediction by Kanamori et al. [12] that the resonance between a horizontal planar pressure source (e.g. coseismic ground movement) and vertically propagating atmospheric acoustic wave occurs at a period of 275 seconds (this is also the acoustic cut-off period; only those with periods shorter than that can propagate as acoustic waves). In the upper panels of Fig. 3, we compare the polynomial fit residuals (dark-gray curves) for satellites 13 (site $\mathrm{G}$ ) and 24 (site C), and band-path filtered (window 3-7 minutes) time series (light-gray curves). The lower panels of Fig. 3 show time-period diagrams obtained by wavelet transformation with a Mexican-hat mother function. They indicate the CID period of 4-5 minutes for satellite 13. Such a period is ambiguous for satellite 24 . This apparent difference reflects the geometry of the satellite 24 line-of-sight that has penetrated both the positively and the negatively perturbed parts in the ionosphere. This will be discussed later in the section 4.1 .

In contrast to Tohoku GPS stations, those in Hokkaido show no clear TEC disturbance signals in spite of similar epicentral distances (upper half, Fig. 2a). For the Kii foreshock (Fig.2c) were also seen similar CID signals, also characterized by positive initial motions and period of 4-5 minutes. Apparent growth of amplitude with time in Fig.2c would rather reflect azimuthal asymmetry of CID propagation. The origin of such directivity will be discussed in the section 4.3 .

\subsection{Travel time diagram}

From the arrival time differences at different points, apparent velocity of CID can be calculated. To obtain accurate arrival times, we applied wavelet transformation using a Mexican hat wavelet tuned to 
retrieve the CID signals (both of the two pulses, see horizontal white lines in Fig.3b bottom) of satellite 24 (Fig.2a, middle). Figure 4 shows wavelet-transformed time series in colors, painted on curves, showing time and along-surface distances between the SIPs of satellite 24 and the center of uplift (Fig. 1a). The curves take various shapes depending on the site-satellite geometry, but the positions of the first peak arrivals (red part) align on a certain line indicating linear relationship between travel times and focal distances.

This "travel-time diagram" demonstrates that (1) propagation speed (the slope of the best-fit line of the first peak arrival times) is $\sim 1 \mathrm{~km} / \mathrm{sec}$, and (2) propagation starts from the center of uplift $\sim 10$ minutes after the earthquake. By fitting a line to the first peaks in Fig.4, we estimated this "delay" as $10.0 \pm 0.1$ minutes and the apparent propagation speed as $1.25 \pm 0.01 \mathrm{~km} /$ second (errors are $1 \sigma$ ). If the propagation occurs $300 \mathrm{~km}$ above the Earth's surface, the estimated speed should be increased by 5 percent. Ducic et al [4] proposed the mechanism of CID that $\left(1^{\text {st }}\right)$ the Rayleigh wave propagates along the Earth's surface and $\left(2^{\text {nd }}\right)$ the ground movement associated with the surface wave passage induces secondary atmospheric wave that propagates upward and disturb ionosphere. In that case, apparent speed of CID should be similar to the Rayleigh wave of this period, say $\sim 3.8 \mathrm{~km} / \mathrm{sec}$. The speed observed here is much slower and similar to the sound velocity in the upper atmosphere (slightly faster than that; wind in the ionosphere is a possibility but needs further studies). We therefore prefer the opposite mechanism that $\left(1^{\text {st }}\right)$ the atmospheric pulse caused by coseismic uplift propagates upward from the focal region, $\left(2^{\text {nd }}\right)$ it is gradually refracted and eventually propagates horizontally at the ionospheric height. Travel time diagrams for satellites 13 and 27, for the Tokachi earthquake, and satellite 15 for the Kii foreshock also suggested similar delays and propagating velocities. The absence of the disturbance signals by the Rayleigh wave in our cases is further discussed in section 4.2 .

The TEC time series of satellite 24 in Tohoku (A-E in Figs. 2a) suggest that the CID starts as a simple positive pulse (Fig.2a site A) and the second pulse emerges as they propagate (Fig.2a sites B-E). Also in the travel time diagram (Fig.4) are seen this second peaks as a yellow band to the right of the first peak. This is not a simple follow-up wave of the first pulse because they propagate more slowly than the first one, namely by $\sim 0.7 \mathrm{~km} / \mathrm{sec}$. This is too slow for a sound velocity at ionospheric heights. Furthermore, this secondary CID seems to have a period which is longer than the acoustic cut-off and is more likely a gravity wave [12]. Gravity waves in the ionosphere were suggested to be as fast as $0.2-0.8 \mathrm{~km} / \mathrm{sec}$ by GPS observations of LSTID, a large-scale traveling disturbance in TEC caused by a gravity wave originating in the auroral oval $[13,14]$. Thus the secondary CID might be a gravity wave excited by partial transformation of the acoustic wave energy somewhere above the epicenter, but its real excitation mechanism is yet to be investigated.

\subsection{Directivity of the propagation}

The CID radiation is not omni-directional as shown by the signal difference between Hokkaido and Tohoku (Fig. 2a). In Fig.4 inset, we isolate the signals of northward propagating CID by taking out GPS sites in Hokkaido. Their colors suggest that the amplitude of the northward CID is smaller than the southward CID by an order of magnitude. To better visualize such directionality, we plot on the map snapshots of wavelet-transformed values (wavelet periods were tuned to best retrieve satellite-specific CID waveforms) at different epochs to see the CID passage (Fig.5). The satellite 24, which was close to zenith, shows clear southwestward along-arc propagation of the CID while northward propagation into Hokkaido is ambiguous (Fig. 5a). Although the observations of satellite 13 were made at low elevations, they provide important information on the early stage of CID (Fig. 5b); they clearly show southward radiation of the CID energy. This is followed by the observations of satellite 27 (Fig. 5c).

Fig. 6a shows similar snapshots for the Kii foreshock. In this case, circular wave front is clearly seen owing to relatively wide azimuthal coverage of the CID observations. The CID amplitudes vary a lot even along the same wave front. Such a variation is shown in Fig. 6b as amplitudes at the epoch 13 minutes after the earthquake ( $3^{\text {rd }}$ panel of Fig. 6a) as a function of azimuths. We can see that the abrupt change in amplitude occurs at azimuths $230^{\circ}-280^{\circ}$, and the amplitude contrast reaches an order of magnitude. Apparent temporal growth of CID amplitudes seen in Fig.2c would be just due to the difference in azimuth of SIPs as seen from the center of uplift. Quantitative discussion on the origin of the directivity is given later in the section 4.3 .

3.4. Disturbance height and thin ionosphere approximation

It is often assumed that the ionospheric disturbance takes place within a thin shell. Ducic et al. [4] 
suggested that its height of 290-300 km maximizes the correlation of disturbance signals between different satellites. From LSTID studies with GEONET, Saito et al. [13] also suggested a local ionospheric height of $\sim 300 \mathrm{~km}$. We can confirm this in Fig. 6a, plotted assuming thin ionosphere as high as $300 \mathrm{~km}$. Because the surface wind velocity on 5 September 2004 was less than $5 \mathrm{~m} / \mathrm{sec}$ (www.jma.go.jp), the center of the disturbance should nearly coincide with the maximum uplift inferred from other geophysical observations. This is the case as seen in Fig. 6a. If we change the ionospheric height by $50 \mathrm{~km}$, the apparent disturbance center shifts by $\sim 100 \mathrm{~km}$ from the center of uplift together with the entire SIPs. The apparent coincidence justifies the assumed height, which nearly corresponds to the maximum electron density (Fig.7a).

Waves obliquely penetrating the ionosphere undergo larger delays (by a factor of $1 / \cos \theta$, where $\theta$ is the zenith angle at the ionosphere). The data plotted in Fig. 5 and 6 are corrected for this factor assuming a thin ionosphere, so that signals of different satellites (i.e. different zenith angles) look similar for the same SIP and epoch. This is, however, not the case, as seen by comparing the two panels, i.e. 13:00 epoch of satellite 24 (Fig. 5a, $1^{\text {st }}$ panel), and 13:30 epoch of satellite 13 (Fig. 5b, $3^{\text {rd }}$ panel). The apparent CID amplitudes of satellite 13 are significantly larger than satellite 24 . This suggests that the CID actually has 3-dimensional structure in the ionosphere of finite thickness.

\section{Discussion}

4.1. Arrival times, waveforms, and relative amplitudes

Sound velocity scales with the square-root of the temperature [12], and the increase of the velocity with altitude bends the ascending acoustic waves downward. We performed a simple ray tracing in the atmosphere, following Calais et al. [10], with height-dependent sound velocity shown in Fig. 7a. Time required for the vertically-propagating wave to reach the ionosphere is about ten minutes, which is consistent with our result (section 3.2). Fig. $7 \mathrm{~b}$ indicates that wave emitted toward the elevation $70^{\circ}-90^{\circ}$ (zenith angle $0^{\circ}-20^{\circ}$ ) reach the ionospheric heights, and those with lower launch angle come back to the earth without reaching that height. A velocity minimum at the height of $\sim 100 \mathrm{~km}$, caused by the temperature inversion, traps waves and let them propagate horizontally at this height. Such a ducted wave, however, is too low to disturb the ionosphere.

We calculated the TEC change by integrating the disturbance of each of the ray paths at its intersection with the line-of-sight, a technique similar to Calais et al. [10]. In Fig. 8 we plot such synthesized TEC changes for satellites 13 and 24 at every 30 second interval, of two GPS stations for each satellite. In order to avoid directivity effects, we selected GPS stations with SIPs in almost the same azimuth (SW of the epicenter, see Fig. 5a,b). We assumed a bipolar source function composed of a positive part and a smaller negative part (Fig.8 middle). Its amplitude is scaled to match with the observations, so only the relative amplitude differences, waveforms, and arrival times are meaningful here.

The simulation results show that (1) CID for satellite 13 is several times as large as those for satellite 24, (2) CID waveforms of satellite 13 well preserve the shape of the source function while the negative parts are blurred for satellite 24, and (3) CID occurs 11-12 and 13-14 minutes after the earthquake for the satellite 13 and 24, respectively. All these features are consistent with the observed CID signals. The features (1) and (2) reflect the geometric difference between the line-of-sights and the disturbance wave fronts, i.e. the satellite 24 line-of-sight penetrates both the high and low electron density regions in the perturbed ionosphere while that of satellite 13 is more or less parallel with the front. This is responsible for the apparent difference of CID waveforms and amplitudes seen in Fig. 2a,b. Positive initial motions (TEC increase) is understood as the manifestation of the propagation of a compressive atmospheric pulse generated by the uplift of the sea surface. This would reverse for normal-fault earthquakes.

\subsection{Absence of the Rayleigh wave signatures}

Next we discuss why the ionospheric disturbances caused by the Rayleigh surface wave, as observed in southern California for the 2002 Denali Earthquake [4], are not seen in this study. Indeed, the velocity difference between the Rayleigh wave $(\sim 3.8 \mathrm{~km} / \mathrm{sec})$ and the sound wave $(\sim 1.0 \mathrm{~km} / \mathrm{sec})$ suggests that they are already apart by $\sim 5$ minutes at $\sim 400 \mathrm{~km}$ from the epicenter. However, we cannot sea significant disturbances in the time series in Fig. 2a preceding the main CID.

In seismograms, surface wave and $\mathrm{S}$ wave signatures are not well distinguishable up to, say $20^{\circ}$ from the epicenter because they arrive almost simultaneously. Because the vertical ground movements close to epicenter are the mixtures of the two waves with different velocities and periods, they may not be 
spatially coherent enough to disturb the atmosphere effectively. On the other hand, CIDs of acoustic wave origin (as well as seismic S waves) decay more rapidly than surface waves because they propagate in 3-dimensional space. At a sufficient distance from the epicenter, acoustic origin CID may have significantly decayed leaving only the Rayleigh wave signatures observable in TEC time series. This might be the Denali case [4], where GPS points are $>2000 \mathrm{~km}$ apart from the epicenter, and other cases reported recently using Doppler sounding [15]

\subsection{Origin of the directivity}

After mine blasts in North America, Calais et al. [10] noticed the north-south asymmetric ionospheric responses, and suggested two possibilities, (1) the interaction with the local geomagnetic field, and (2) azimuthal difference in energy emission in terms of seismic wave. For CID, interferences between waves of opposite phases from uplifted and subsided regions might cause such asymmetry. Then, the largest attenuation would occur along the direction perpendicular to the line connecting the two regions. This is, however, not the case. Generally speaking, thrust earthquakes induce more uplift than subsidence (Fig.1), which would look like a single uplift from a distance. In this study, we pursue the possibility (1) considering electromagnetic forces acting on the moving particles during the acoustic wave propagation.

Fig. $7 \mathrm{~b}$ shows direction of geomagnetic field in the focal region of the Tokachi earthquake, dipping $\sim 56^{\circ}$ from horizon [16]. The field would hence look like $\boldsymbol{H}$ and $\boldsymbol{H}^{\prime}$ for southward and northward propagating waves, respectively. In acoustic waves, particles oscillate in the direction of propagation, and the magnetic field exerts the Lorentz Force on charged particles, and hampers their movements perpendicular to the field. Hooke [17] formulated the behavior of the movements of ionized particles surrounded by moving neutral particles. Ion movement is influenced both by the neutral atmosphere movements and ambient geomagnetic field directions. Their relative importance is determined by the ratio of the ion-neutral collision frequency to the ion gyro-frequency. This ratio is small enough at the $\mathrm{F}$ layer height (where the observed CID exists), and the ion moves only in the direction of the magnetic field irrespectively of the neutral particle movements (equation 2 of [17]).

The front of the northward CID becomes parallel with $\boldsymbol{H}^{\prime}$ at some instant between 10 and 15 minutes after the earthquake (Fig.7b), when the Lorentz force prohibits its further propagation. On the other hand, southward propagating wave front never gets parallel with $\boldsymbol{H}$, and the CID survives until the ionospheric heights. Using the ray tracing results given in Fig. $7 \mathrm{~b}$, we tried to reproduce the "beam pattern" shown in Fig. 6b. The magnetic inclination and declination is $47^{\circ}$ and $-6^{\circ}$, respectively, in the Kii foreshock area [16]. We calculated the inner products of the propagation directions (i.e. the direction of the neutral particle motion) and the magnetic fields, multiplied by electron density (Fig.7a), and integrated them along the three ray paths with zenith angles $18^{\circ}, 19^{\circ}$, and $20^{\circ}$, which are the main carriers of the CID. A large integrated value means a larger chance of survival of the CID. The obtained beam patterns are shown in Fig. 6b. In spite of some differences in pattern among ray paths, they show steep changes around azimuths of $230^{\circ}-280^{\circ}$, which is consistent with the observations.

The model presented here predicts different beam patterns in other regions of the world. For example, the opposite would occur in the mid-latitude of the southern hemisphere (i.e. attenuation of southward propagation). In the polar region ( $\boldsymbol{H}$ is vertical), stronger attenuation would occur for all the directions while two (north- and southward) beams would survive in equatorial regions ( $\boldsymbol{H}$ is horizontal). Future studies of CIDs for such earthquakes would further substantiate our model.

\section{Acknowledgements}

We thank two reviewers whose comments significantly improved the quality of the paper. Discussions with Akinori Saito (Kyoto Univ.), Takuya Tsugawa (Nagoya Univ.), Shingo Watada (Univ. Tokyo), and Hirotomo Noda (NAO) were fruitful.

\section{References}

[1] P.C. Yuen, P.F. Weaver, R.K. Suzuki, Continuous, traveling coupling between seismic waves and the ionosphere evident in May 1968 Japan earthquake data, J. Geophys. Res., 74 (1969) 2256-2264.

[2] T. Tanaka, T. Ichinose, T. Okusawa, T. Shibata, Y. Sato, C. Nagasawa, T. Ogawa, HF Doppler observations of acoustic wave exhibited by the Urakawa-Oki earthquake on 21 March 1982, J. Atmos. Terr. Phys. 46 (1984) 233-245.

[3] E. Calais, J.B. Minster, GPS detection of ionospheric perturbations following the January 17, 1994, 
Northridge earthquake, Geophys. Res. Lett. 22 (1995) 1045-1048.

[4] V. Ducic, J. Artru, P. Longnonné, Ionospheirc remote sensing of the Denali Earthquake Rayleigh surface wave, Geophys. Res. Lett. 30 (2003) 1951,doi:10.1029/2003GL017812.

[5] K. Heki, Dense GPS array as a new sensor for seasonal changes of surface loads, in: R.S.J. Sparks and C.J. Hawkesworth (Ed.), The State of the Planet: Frontiers and Challenges in Geophysics, AGU Geophys. Monograph, 150 (2004) 177-196.

[6] K. Heki, Secular, transient and seasonal crustal deformation and its geophysical implications, in: T. Dixon and C. Moore (Ed.), Seismogenic Zone of Subduction Thrust Faults, Columbia University Press (2005) in press

[7] Y. Okada, Internal deformation due to shear and tensile faults in a half-space, Bull. Seism. Soc. Am. 82 (1992) 1018-1040.

[8] Y. Tanioka, K. Hirata, R. Hino, T. Kanazawa, Slip distribution of the 2003 Tokachi-oki earthquake estimated from tsunami waveform inversion, Earth Planets and Space 56 (2004) 373-376.

[9] Earthquake Research Committee, On the seismic activity of southeast off-Kii-Peninsula, Documents in the 131th Earthq. Res. Committee Meeting, http://www.jishin.go.jp, (2004) (in Japanese)

[10] E. Calais, J.B. Minster, M.A. Hofton, M.A. H. Hedlin, Ionospheric signature of surface mine blasts from Global Positioning System measurements, Geophys. J. Int. 132 (1998) 191-202.

[11] E.L. Afraimovich, N.P. Perevalova, A.V. Plotnikov A.M. Uralov, The shock-acoustic waves generated by earthquakes, Ann. Geophys. 19 (2001) 395-409.

[12] H. Kanamori, J. Mori, D.G. Harkrider, Excitation of atmospheric oscillations by volcanic eruptions, J. Geophys. Res. 99 (1994) 21,947-21,961.

[13] A. Saito, S. Fukao, S. Miyazaki, High resolution mapping of TEC perturbations with the GSI GPS network over Japan, Geophys. Res. Lett. 25 (1998) 3079-3082.

[14] T. Tsugawa, A. Saito, Y. Otsuka, A statistical study of large-scale traveling ionospheric disturbances using the GPS network in Japan, J. Geophys. Res. 109 (2004) doi:10.1029/2003JA010302.

[15] J. Artru, T. Farges, P. Lognonné, Acoustic waves generated from seismic surface waves: propagation properties determined from Doppler sounding observations and normal-mode modelling, Geophys. J. Int. 158 (2004) 1067-1077.

[16] NAO (National Astronomical Observatory), Chronological Scientific Tables 2004, Maruzen, Tokyo, 2003, $945 \mathrm{pp}$.

[17] W.H. Hooke, The ionospheric response to internal gravity waves 1. The F2 region response, J. Geophys. Res. 75 (1970) 5535-5544. 


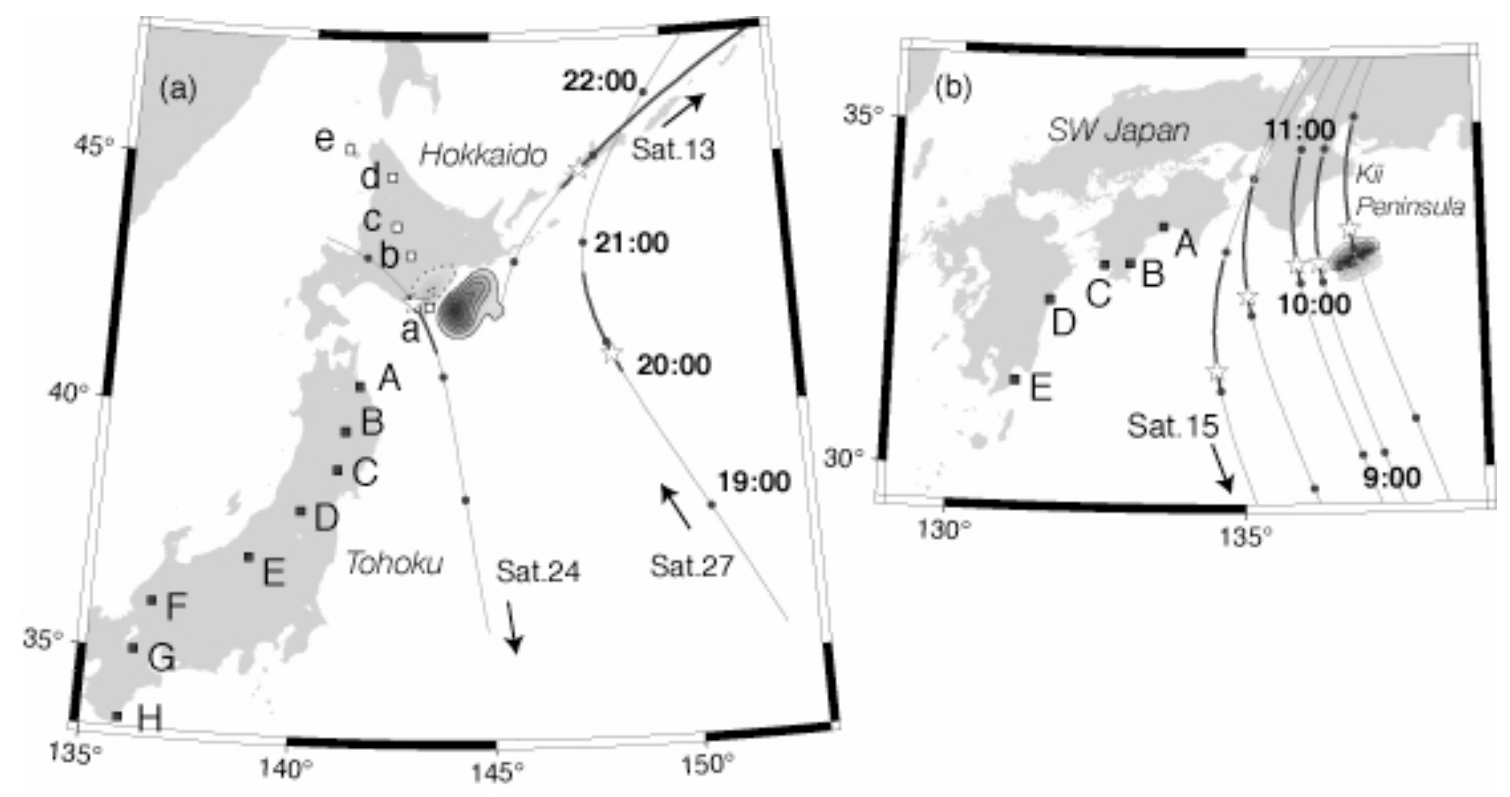

Fig. 1. Vertical coseismic crustal movements for the Tokachi earthquake (a) and the Kii foreshock (b) are shown as contours with intervals of $10 \mathrm{~cm}$. The maximum uplifts (solid line contours) are $\sim 70 \mathrm{~cm}$ for both earthquakes but occurred over a larger area in the Tokachi case. Squares in the maps show GPS stations whose TEC signals are plotted in Fig. 2. SIP trajectories, calculated for the GPS station "a", are shown as solid curves for three satellites 13, 24 and 27 in (a). SIP trajectories for satellite 15, as seen at the five GPS stations "A" to "E", are plotted for the Kii case (b), which shows that SIP tracks for different stations are approximated by simple translations. Solid circles on the SIP tracks are time stamps for 19:00, 20:00, 21:00, and 22:00 UT for (a) and 9:00, 10:00, and 11:00 UT for (b). Thick parts in the tracks correspond to time windows shown in Fig. 2, and open stars are time stamps of the earthquake occurrences.
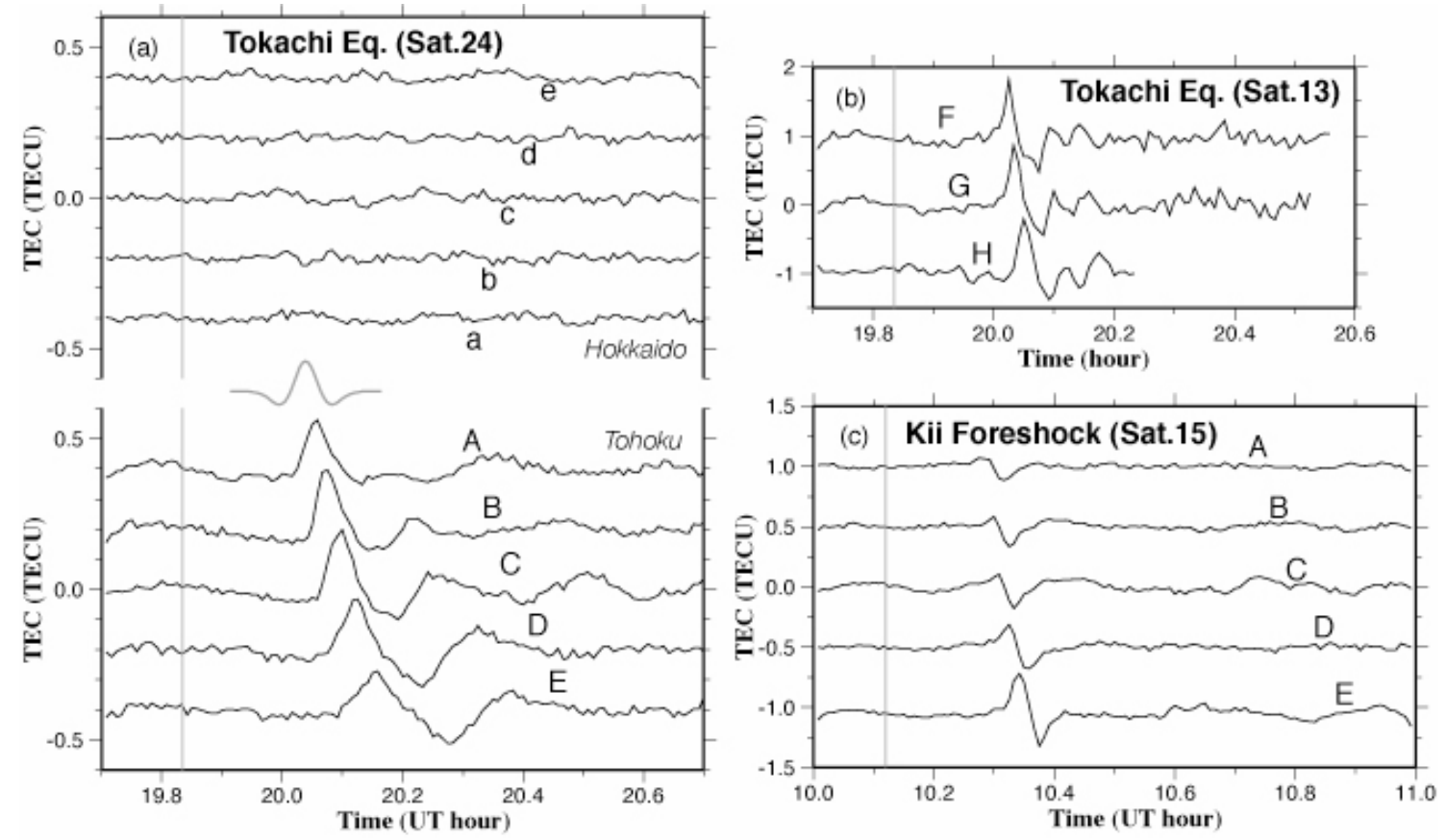
Fig. 2. (previous page) TEC time series for satellite 24, obtained as residuals from the polynomials (degree 6) fit to the raw time series, at five sites in Hokkaido ("a" to "e") and in Tohoku ("A" to "E") over a one-hour interval 19.7-20.7 UT (a). The time series for satellite 13 at three sites ("F" to "H") in SW Japan are also shown (b) (data terminate before the end of the window as the satellite elevation becomes lower than $15^{\circ}$ ). Gray vertical lines denote the time of earthquake occurrence, i.e. $\sim 19: 50$ (a, b) and 10:07 (c). Similar plots with satellite 15 are given for sites "A" to "E" (Fig.1b) 10.0-11.0 UT for the Kii foreshock (c). A Mexican hut wavelet in (a) is used to draw the travel time diagram of Fig. 4.
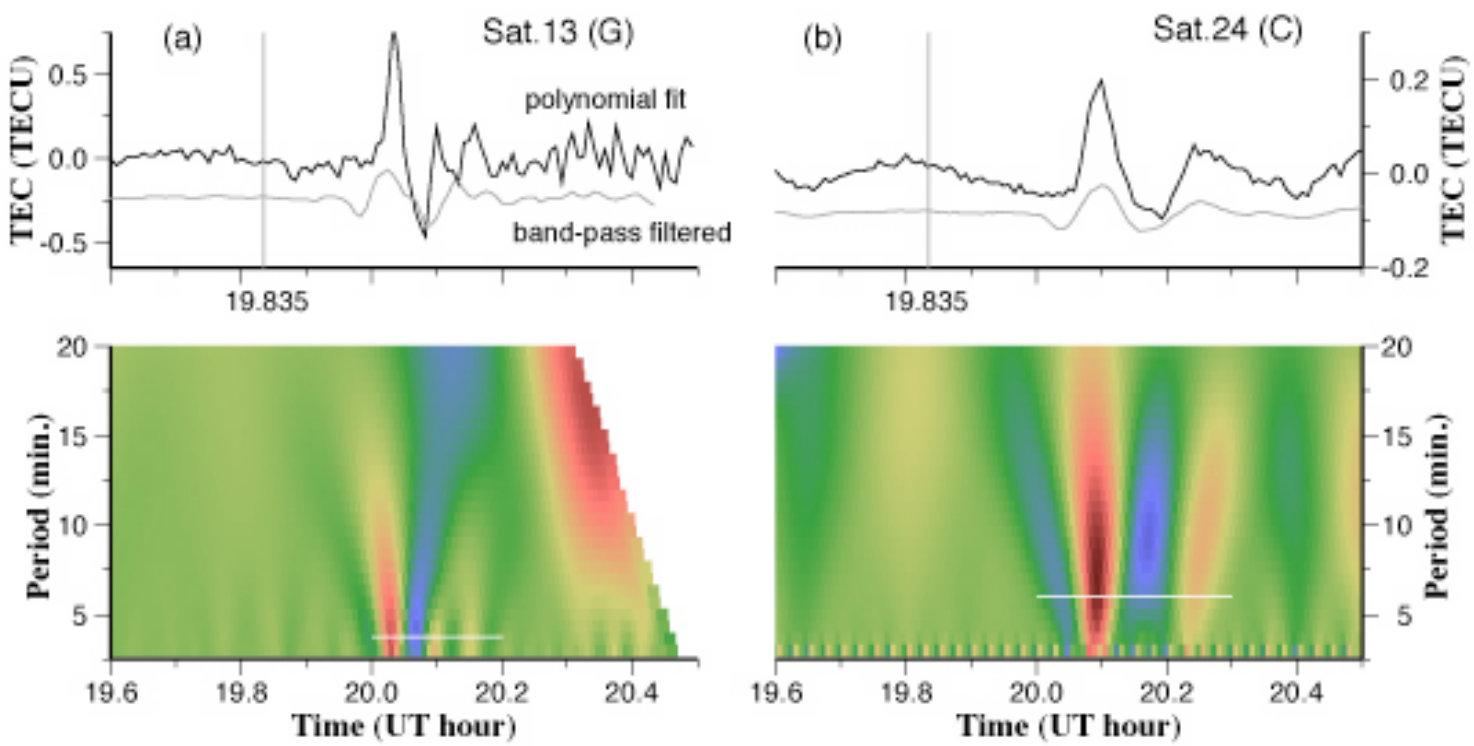

Fig. 3. Two kinds of time series (dark gray: residual of the polynomial fit, light gray: band-pass filtered with a window of 3-7 minutes) are compared for satellites (a) 13 (site G in Fig. 1a), and (b) 24 (site C in Fig. 1a). Below them are drawn period-time diagrams obtained by wavelet analyses. The period of the main CID signal (at 20.03) is 4-5 minutes for satellite 13 (a) but is somewhat longer for satellite 24 (b). In addition to the main peak at $\sim 20.1$, the satellite 24 data have a secondary peak at $\sim 20.25$, which propagates a little more slowly (see Fig.4). White horizontal lines in the bottom panels indicate the period of the wavelet used to draw Figs. 4 and 5a,b.

Fig. 4. (next page) Travel time diagram of CID obtained for satellite 24 after the Tokachi earthquake. Distances are measured from the center of the uplift region (Fig. 1a) rather than the seismological epicenter. One curve corresponds to one GPS station. Colors show CID amplitudes obtained by wavelet transformation (mother function shown in Fig. 2a). The arrivals of the main CID are shown by vivid red color indicating the amplitude 0.1-0.2 TECU. The arrivals of the secondary peak are shown by a yellow color band 0.1-0.2 hours after the first one. Their apparent velocities are $\sim 1.2 \mathrm{~km} / \mathrm{sec}$ (first peak) and $\sim 0.7 \mathrm{~km} / \mathrm{sec}$ (second peak). In the inset are plotted only data from GPS stations in Hokkaido. The same color scheme is used in Figs. 3, 5, and 6. 


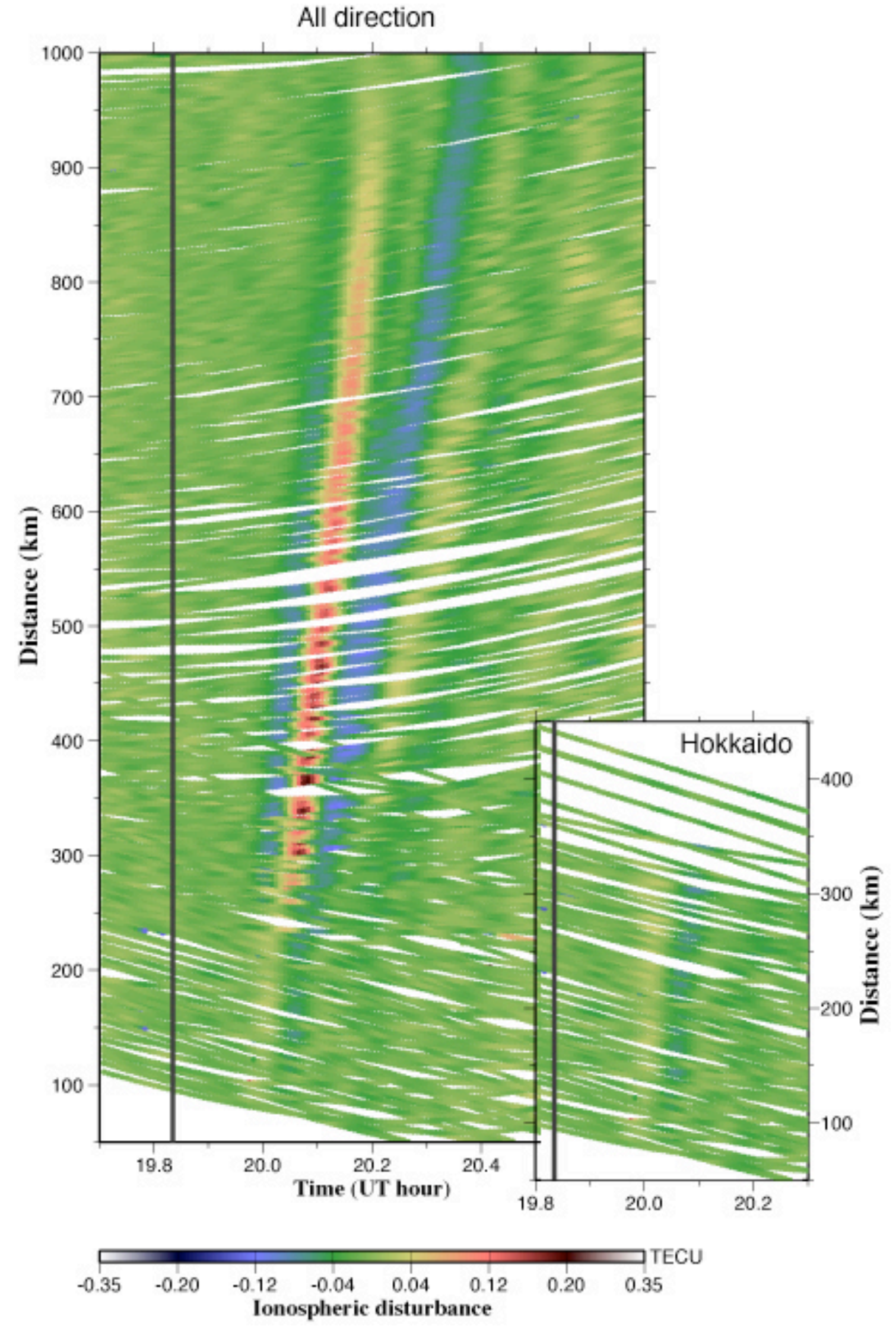




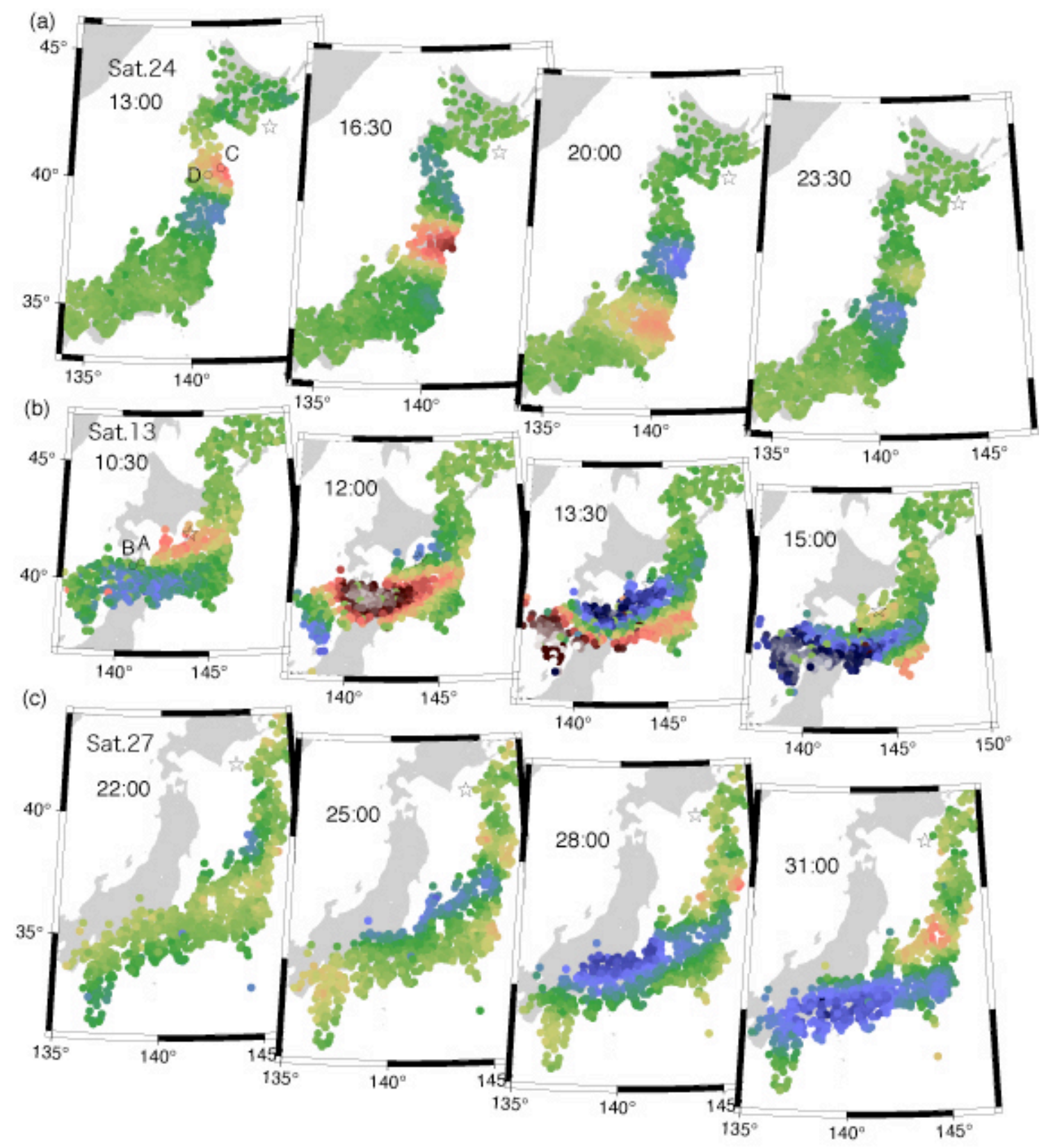

Fig. 5. Snapshots of the propagating CID at four epochs for satellites 24 (a), 13 (b), and 27 (c) after the Tokachi earthquake obtained by wavelet transformation. The open star indicates the point of maximum coseismic uplift. Epochs are indicated as the time (min:sec) after the earthquake for each panel. Absence of the northward CID is clear in (a) and (b). In the first epoch panels of (a) and (b), we show the SIPs ("A", "B" for satellite 13 and "C", "D" for satellite 24) of GPS stations whose time series are plotted in Fig. 8. 


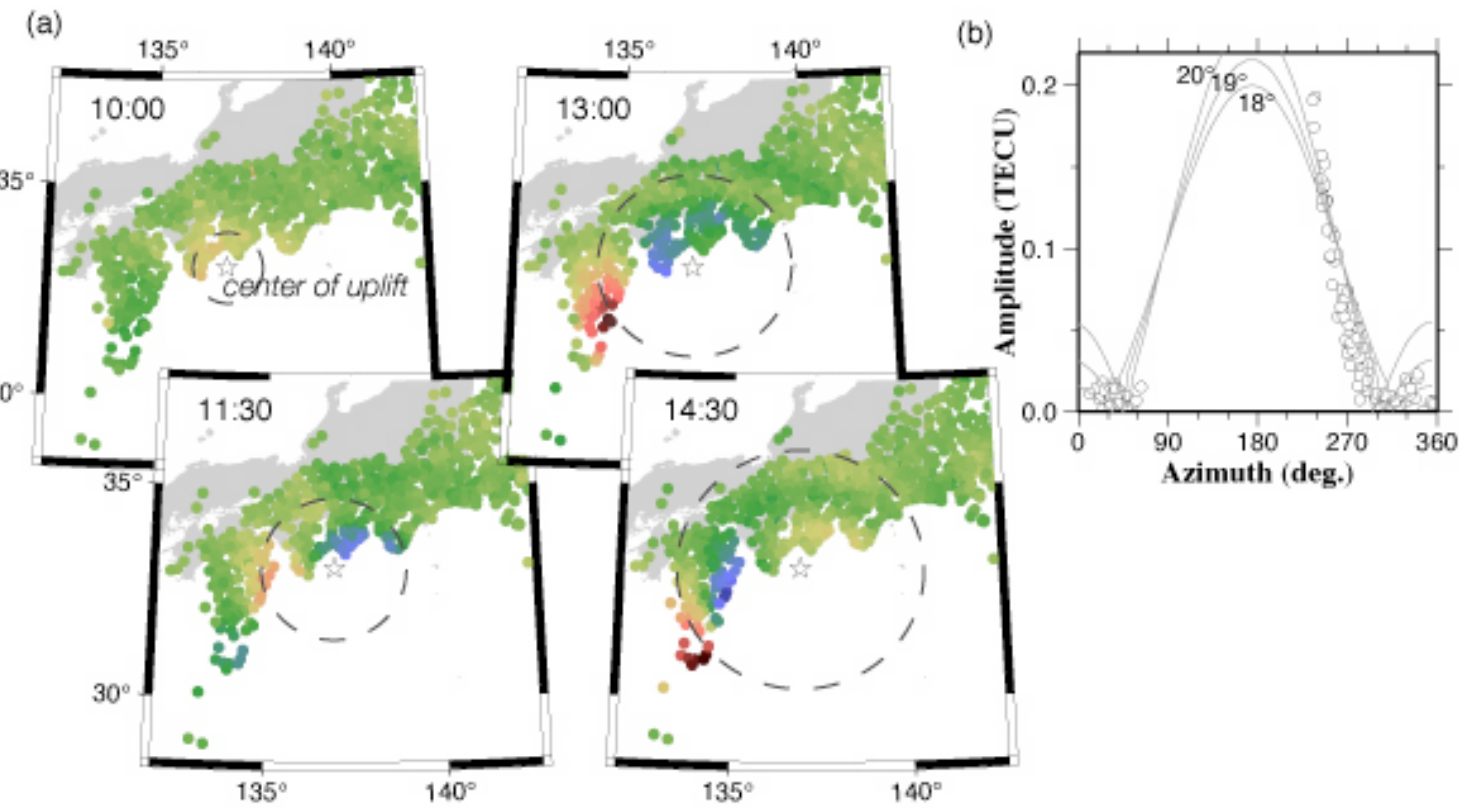

Fig.6. Snapshots at four epochs of propagating CID observed with satellite 15 after the Kii foreshock. The open star indicates the point of maximum coseismic uplift. We can see circular wave front with the center nearly coincident with the open star. CID amplitudes at the 13:00 epoch, of points whose SIPs are under the wave front, were plotted as a function of azimuths (b). In (b), beam patterns predicted by numerical studies for ray paths with zenith angles $18^{\circ}, 19^{\circ}$, and $20^{\circ}$ are shown as gray curves.

(a)

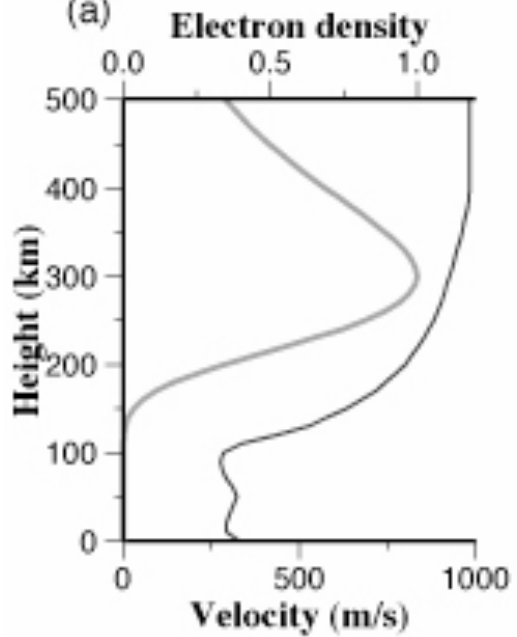

(b)

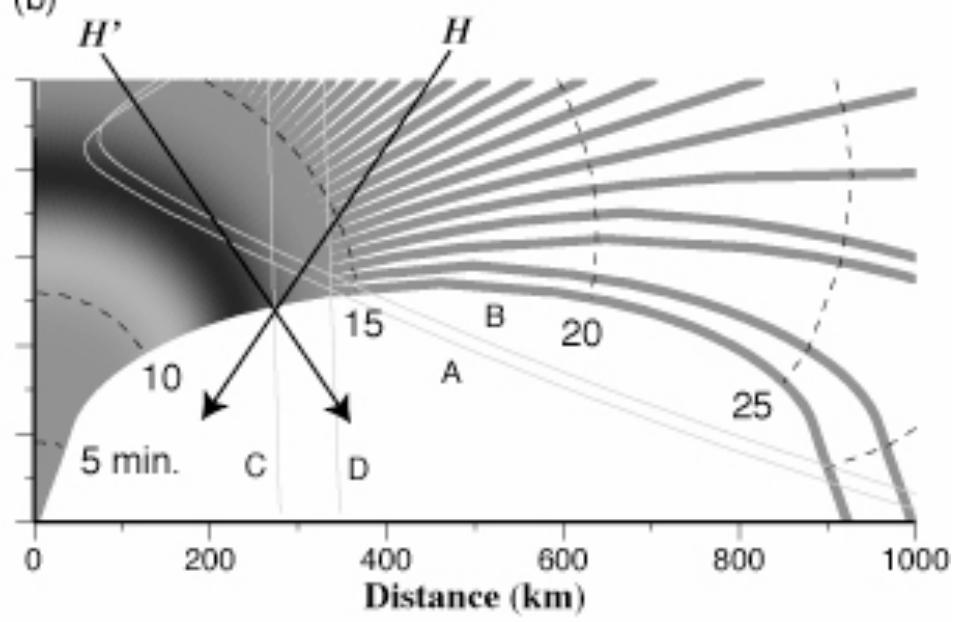

Fig.7. Relative electron density (light gray) and velocity of acoustic wave (dark gray) are plotted as functions of height [10] (a). Ray tracing of acoustic waves for zenith angles 0-20 degrees is done assuming this velocity profile (b). Equal-time contours are shown every five minutes after the earthquake with broken curves. Gray scales shown along the ray paths indicate the position of the source function (Fig. 8) at the epoch $\sim 11$ minutes (when the positive peak overlaps with the line "A"). Gray curves are the line-of-sights connecting the satellites 13, 24 and four GPS stations (their SIPs are given in Fig. 5). Their observed and synthesized TEC time series are given in Fig. 8 . These line-of-sights are linear in 3-dimensional space, but have apparent curvatures here because we took the epicentral distance as the horizontal axis. Geomagnetic field inclination is $\sim 56^{\circ}$ in the Tokachi earthquake epicentral area ( $\boldsymbol{H}$ and $\left.\boldsymbol{H}^{\prime}\right)$. 


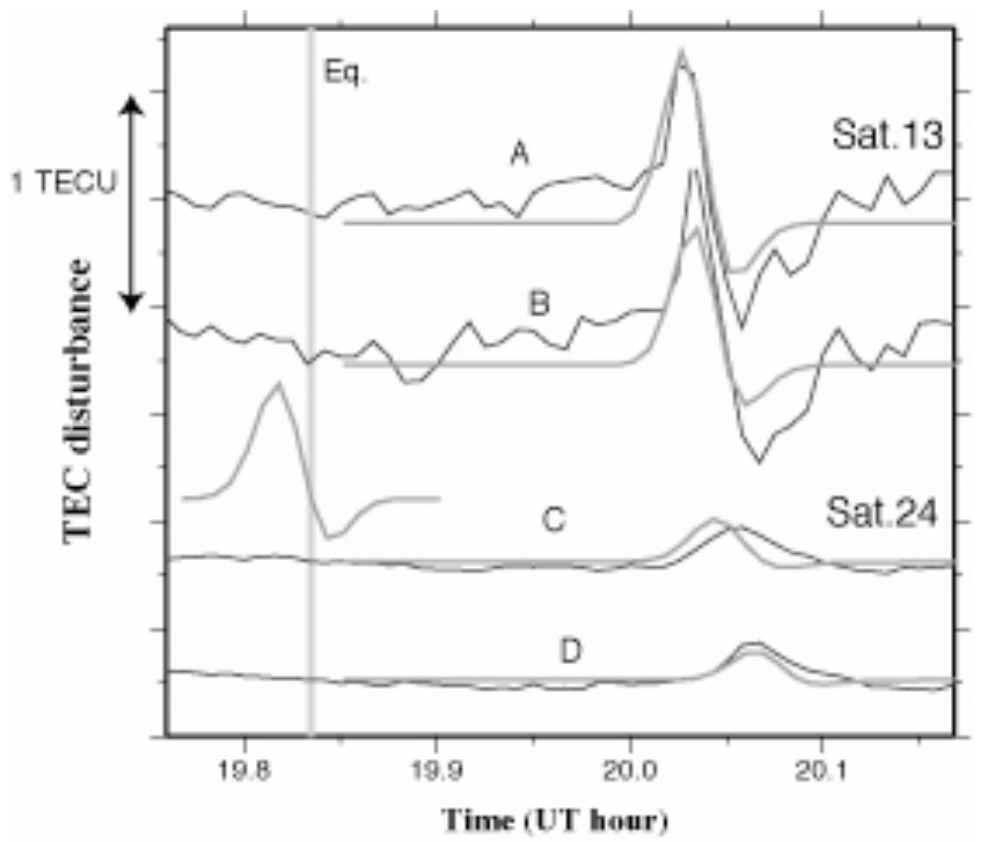

Fig.8. Observed (dark gray, residuals of the polynomial fit) and synthesized (light gray, based on the ray tracing in Fig. 7) TEC time series for satellites 13 ("A", "B") and 24 ("C", "D") at four GPS stations with SIPs located southwest of the epicenter (Fig. 5). In the middle, we show the assumed source function. The observed arrival times, waveforms, and relative amplitudes are consistent with the synthesized curves. 